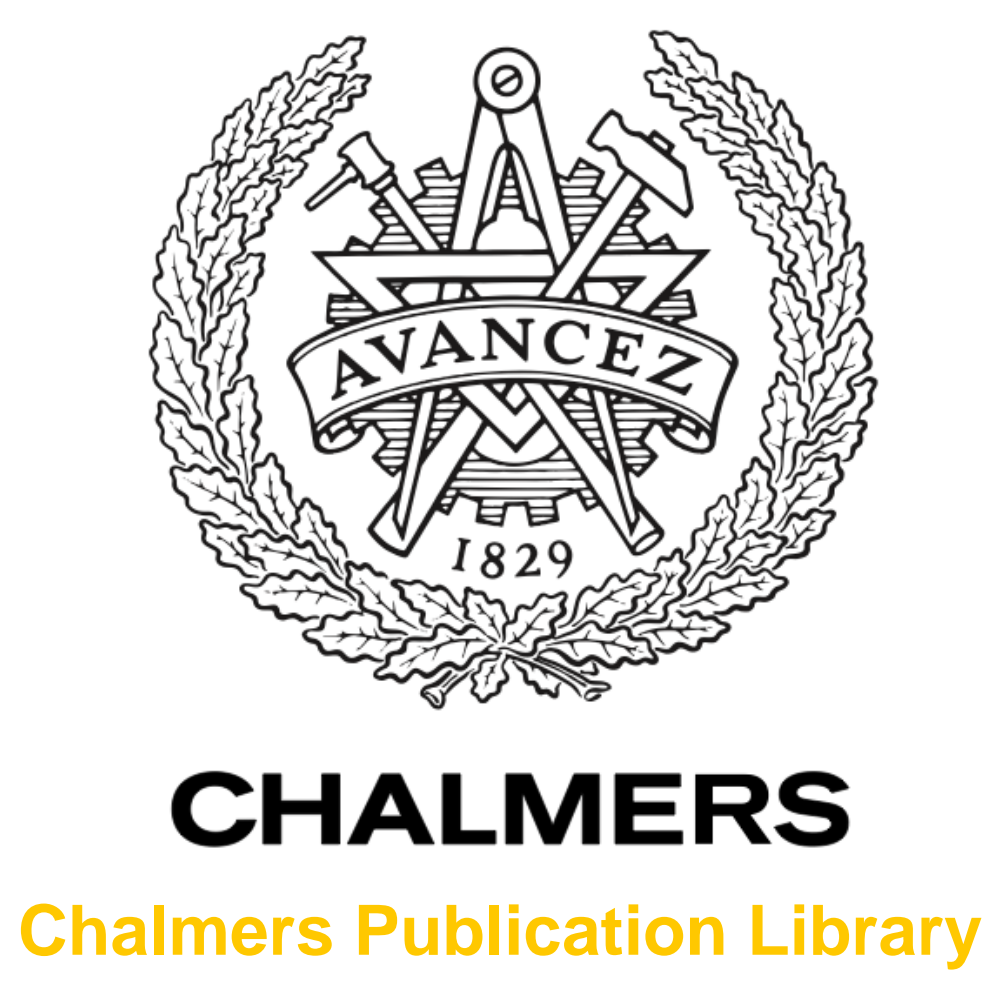

\title{
Performance Evaluation of Multilevel Converter based Cell Balancer with Reciprocating Air Flow
}

This document has been downloaded from Chalmers Publication Library (CPL). It is the author's version of a work that was accepted for publication in:

\section{IEEE Vehicle Power and Propulsion Conference}

\author{
Citation for the published paper: \\ Altaf, F. ; Johannesson, L. ; Egardt, B. (2012) "Performance Evaluation of Multilevel \\ Converter based Cell Balancer with Reciprocating Air Flow". IEEE Vehicle Power and \\ Propulsion Conference pp. 706-713.
}

http://dx.doi.org/10.1109/VPPC.2012.6422634

Downloaded from: http://publications.lib.chalmers.se/publication/166614

Notice: Changes introduced as a result of publishing processes such as copy-editing and formatting may not be reflected in this document. For a definitive version of this work, please refer to the published source. Please note that access to the published version might require a subscription.

\footnotetext{
Chalmers Publication Library (CPL) offers the possibility of retrieving research publications produced at Chalmers University of Technology. It covers all types of publications: articles, dissertations, licentiate theses, masters theses, conference papers, reports etc. Since 2006 it is the official tool for Chalmers official publication statistics. To ensure that Chalmers research results are disseminated as widely as possible, an Open Access Policy has been adopted.

The CPL service is administrated and maintained by Chalmers Library.
} 


\title{
Performance Evaluation of Multilevel Converter based Cell Balancer with Reciprocating Air Flow
}

\author{
Faisal Altaf, Lars Johannesson and Bo Egardt
}

\begin{abstract}
The modeling and design of an active battery cell balancing system using Multilevel Converter (MLC) for EV/HEV/PHEV is studied under unidirectional as well as reciprocating air flow. The MLC allows to independently switch ON/OFF each battery cell in a battery pack. The optimal policy (OP ) exploiting this extra degree-of-freedom can achieve both temperature and state-of-charge (SoC) balancing among the cells. The $O P$ is calculated as the solution to a convex optimization problem based on the assumption of perfect state information and future driving. This study has shown that $O P$ gives significant benefit in terms of reduction in temperature and SoC deviations, especially under parameter variations, compared to uniformly using all the cells. It is also shown that using reciprocating flow for OP gives no significant benefit. Thus, reciprocating flow is redundant for MLC-based active cell balancing system when operated using $O P$.
\end{abstract}

\section{INTRODUCTION}

Driven by the needs to reduce the dependence of fossil fuels and the environmental impact of transportation there has in recent years been an increasing interest in the electrification of vehicles. The still relatively low specific energy and the high cost of available battery technology means that Hybrid Electric Vehicles (HEVs) and Plug-in Hybrid Electric Vehicles (PHEVs) are in the short term horizon more likely to reach a wide spread impact on the market than pure Electric Vehicles (EVs). Common to both (P)HEVs and EVs is that the battery is one of the most expensive components in the powertrain, contributing largely to the total vehicle cost. As a result, the battery lifetime is an important factor for the success of (P)HEVs and EVs.

The battery pack (BP) is built from a large number of small cells connected in series and parallel to meet both the traction power demand and electric range requirement. The Depth-of-Discharge (DoD) is one of the most important factors that determines the degradation of the battery cells, see [1], [2], and [3]. To ensure uniform life-time of the cells it is therefore important to utilize each cell so that the Stateof-Charge (SoC) and respectively the DoD, remains almost balanced in all cells of the battery pack. Another factor that strongly influences the lifetime is the cell temperature; hotter cells degrade more quickly than colder cells, see [4], [5], [6], and [7]. Therefore, even a few overheated cells may result in shortening the lifetime of the whole battery pack. Temperature imbalance between cells is mainly caused by variation in internal resistances, temperature gradient in coolant due to convective heat transfer alongside the battery pack, and nonuniform external local thermal disturbances, see [7] and [8]. It

The authors are with Chalmers University of Technology, Department of Signals and Systems, 41296 Gothenburg Sweden. E-mails: \{faisal.altaf, larsjo, bo.egardt\}@chalmers.se. The work of the authors was supported by the Chalmers Energy Initiative. has been reported that the lifetime of Li-Ion cell is reduced by two months for each degree of temperature rise in an operating temperature range of 30 to $40^{\circ} \mathrm{C}$, see [9], and above $40^{\circ} \mathrm{C}$ it decreases drastically.

Hence, the Battery Management Unit (BMU) should ideally be able to both balance the SoC of the cells and keep the temperature differences between the cells less than $5^{\circ} \mathrm{C}$ with a maximum temperature below $40^{\circ} \mathrm{C}$, see [5]. Forced convection cooling is normally used to keep the batteries within recommended operating temperature range but suffers from temperature gradient problem due to convective heat transfer along the coolant fluid stream. The reciprocating airflow $(R F)$ has also been proposed in [4] and [8] to improve temperature uniformity in the battery system, but in our current study it is shown that in the presence of parameter variation and local disturbances, cells can still suffer from non-uniform local heated spots. In addition to forced cooling of the battery system, there are several active and passive cell balancing schemes. These are based on various topologies of switched capacitive and resistive circuits, see for example [10], [11], [12], and [13]. The main idea behind all active balancing schemes is to transfer the charge from cells having higher SoC to cells having lower SoC through, for example, switched capacitors which act as intermediate storage banks.

In recent years cascaded multi-level converters (MLC), see [14] and [15], have been thoroughly investigated and discussed for the drive of electric motor in HEVs, see [16] and [17]. The MLC consists of $n$ cascaded H-bridges with an isolated battery cell for each $\mathrm{H}$-bridge. The combination of an $\mathrm{H}$-bridge and a battery cell is called here a Power Cell (PC). The MLC, other than reducing total harmonic distortion (THD) in generated waveform for the electric machine, also offers an additional advantage of extra degree of freedom to generate the load voltages.

In most of these motor drive applications of MLCs, the usual strategy is to use Phase Shifted Pulse Width Modulation (PSPWM) technique to achieve uniform use of cascaded cells, see [14] and [15]. However, since the cells are not identical and operate in different conditions, SoC and thermal imbalance cannot be avoided. In this article, the PS-PWM scheme is denoted as UDCO (Uniform Duty Cycle Operation) whereas the optimal scheme to control MLC is denoted as OP (Optimal Policy).

In [18], the potential benefit of using the MLC to balance both the SoC and the temperature among the battery cells under unidirectional flow (UF) has been thoroughly investigated and compared to UDCO. The main contribution of the current article is to do the similar investigation for $O P$ under $\mathrm{RF}$ and then compare the results with those of OP under UF . The optimal control policy is calculated as the solution to 
a convex optimization problem based on the assumption of perfect information of the SoC and temperature of each cell as well as of the future driving. The main research task is to investigate any potential benefits of $R F$ for $O P$ based active cell balancing. The second task is to investigate if $O P$ gives a significant benefit compared to UDCO under both $\mathrm{UF}$ and $\mathrm{RF}$. At this initial stage, the evaluation is carried out through simulations. For simplicity, in this early study the electric machine is assumed to be a DC machine and the cells are modeled by resistive circuits. Moreover, the simulation study is focused only on an air-cooled battery sub-module (BSM ) with 5 series-connected cells. The coolant flow is assumed to be laminar with known inlet temperature and speed. The resistance of the thermally exposed downstream cell is assumed to be almost $50 \%$ higher than others to carefully examine the performance of the UDCO and OP under both UF and RF. Another important contribution of this article is the detailed derivation of a state-space electro-thermal model of a battery submodule under the switching action of an MLC under $\mathrm{RF}$. The model is formulated in the context of battery management and optimization. It is pertinent to mention here that though, for the sake of completion, the model with three electrical states of a battery cell have been derived but inside the optimization, some assumptions are made to simplify the problem.

The paper is organized as follows. Section II gives an overview of basic function of MLC. The detailed electrothermal modeling of battery sub-module under the switching action of MLC is given in Section III. Section IV defines the optimization problem and discusses the numerical solution method. The simulation results and comparison between $\mathrm{OP}$ and UDCO scheme under both UF and RF is given in Section V, and conclusions are highlighted in Section VI.

\section{Multi-Level Converters Overview}

In contrast to two voltage-level converters, consisting of a single large battery connected with a single H-bridge (HB), the MLC consists of many series connected Power Cells (PC) where each PC contains an H-bridge and the independent battery cell as shown in Figure 1. The H-bridge is a switch mode dc-dc power converter, see [19], that produces a four-quadrant controllable dc output using four switches $S_{i 1}, S_{i 2}, \bar{S}_{i 1}, \bar{S}_{i 2}$ as shown in Figure 1. Therefore, depending on which switch pair is turned-on, three modes of operation can be defined for each $\mathrm{PC}_{i}$. In Mode-1 $v_{L i}>0$, in Mode-2 $v_{L i}<0$ and in Mode-3 $v_{L i}=0$. To model these three modes of operation, let's define $S_{i j}=1$ for ON-State and $S_{i j}=0$ for OFF-State of switch $S_{i j}$ where ' $i$ ' corresponds to $\mathrm{PC}_{i}$ and $j \in\{1,2\}$. Now the switching function $s_{i}(t)$ for a Cell $_{i}$ can be defined by $s_{i}(t)=\left(S_{i 1}-S_{i 2}\right) \in\{1,-1,0\}$ corresponding to Mode-1, Mode-2 and Mode-3 respectively. The switching vector $s(t)=\left[\begin{array}{llll}s_{1}(t) & s_{2}(t) & \cdots & s_{n}(t)\end{array}\right]^{T}$ contains switching functions for all $n$ PCs inside the MLC. Thus all three modes of H-bridge can be defined in terms of $s_{i}(t)$. Assuming the ideal switch behavior, the ohmic and switching losses can be ignored and, therefore, the input and output of H-bridge, as shown in Figure 1, are related through the switching function $s_{i}(t)$. Thus, the current through Cell $i$

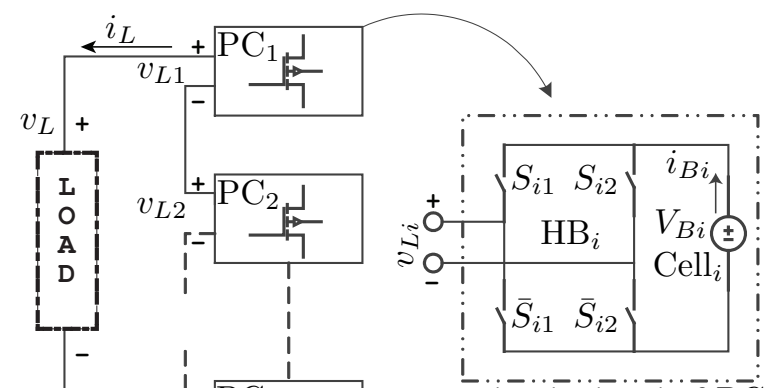

Under the hood of $\overline{\mathrm{PC}}_{i}$

Fig. 1. Block diagram of a single phase cascaded H-bridge multi-leve converter. To avoid the shoot-through problem only one of the switch pairs $\left(S_{i 1}, \bar{S}_{i 2}\right),\left(S_{i 2}, \bar{S}_{i 1}\right),\left(S_{i 1}, S_{i 2}\right)$ or $\left(\bar{S}_{i 1}, \bar{S}_{i 2}\right)$ is allowed to turn-on at a time. The pair $\left(S_{i 1}, \bar{S}_{i 2}\right)$ generates positive $v_{L i}$ and $\left(S_{i 2}, \bar{S}_{i 1}\right)$ gives negative $v_{L i}$ whereas both switch pairs $\left(S_{i 1}, S_{i 2}\right)$ and $\left(\bar{S}_{i 1}, \bar{S}_{i 2}\right)$ gives $v_{L i}=0$.

is given by:

$$
i_{B i}(t)=i_{L}(t) s_{i}(t)
$$

Note that due to the series connection, the same current $i_{L}$ pass through each PC. However, the direction of current passing through the battery $\mathrm{Cell}_{i}$ depends both on the selection of switches and the direction of load current $i_{L}$. Similarly the voltage output from each $\mathrm{PC}_{i}$ is defined by $v_{L i}(t)=$ $V_{B i}(t) s_{i}(t)$ and hence the total voltage output from the MLC can be written as the sum of voltage output from each $\mathrm{PC}_{i}$

$$
v_{L}=\sum_{i=1}^{n} v_{L i}=\sum_{i=1}^{n} V_{B i}(t) s_{i}(t)
$$

with the MLC being able to generate $L=2 n+1$ different voltage levels $\left(v_{L}\right)$.

\section{Modeling of Cell Balancing System With RECIPROCATING AIR FLOW}

The block diagram of the cell balancing system for reciprocating air flow $(R F)$ is shown in Figure 2. In this section, based on the assumption that the load is a DC-machine, first the switching model and then the averaged-state-space model of a power cell is derived and finally the complete state-space model for $n$ power cells is given.

\section{A. Switching Model of a Power Cell}

In this subsection, the electro-thermal model of a switched battery cell under reciprocating air flow is derived. The dynamics of cell temperature depends on many factors like coolant properties, cell material properties, cell placement and battery pack configuration. In [8], the forced-convection cooled battery pack has been modeled using Lumped-capacitance Thermal Model and Flow Network Model (FNM). In that study, the battery pack is configured as $n_{s} S n_{p} P$ which means $n_{p}$ parallel strings (each string is called battery module) with each string having $n_{s}$ cells connected in series. There is a sufficient free space between cells to allow streams of laminar flow of coolant (air). In this paper, the configuration of battery pack used is similar to that in [8] with similar Li-Ion cells and air properties. The various coefficients for thermal and physical 


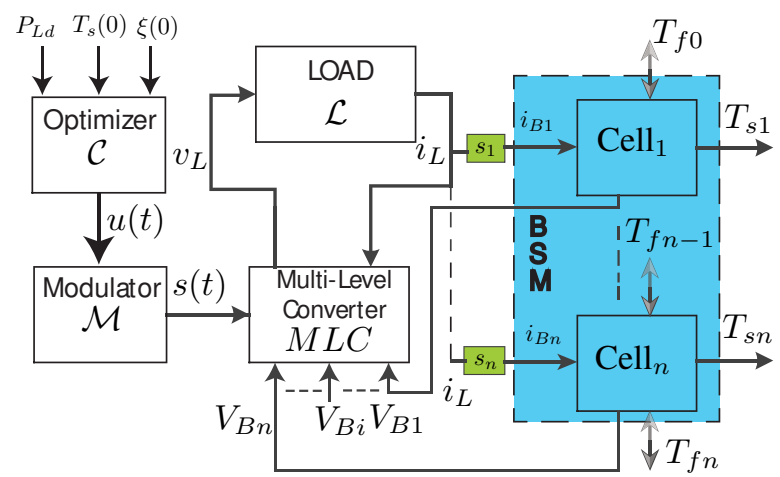

Fig. 2. Conceptual block diagram of battery cell balancing system with reciprocating air flow. $T_{S}(0)$ is a vector containing initial temperature of all cells and $\xi(0)$ is a vector containing initial SoC of all cells, $P_{L d}$ is the demanded power for load with the known voltage and current profile and $T_{f 0}, \cdots, T_{f n}$ represent coolant fluid temperature-nodes. The subsystem inside blue box is a battery submodule (BSM) being balanced and green boxes contain switching functions for the corresponding cell.

properties of cell and air used in this study are given in Table I, see [8] for details. The $C_{N i}[A h]$ is the nominal capacity

TABLE I

DEFINITION OF BATTERY PARAMETERS

\begin{tabular}{lc|lc}
\hline Parameters & Expression & Parameters & Expression \\
\hline$a_{s i}\left[\frac{1}{s}\right]$ & $\left(\frac{1}{C_{s i} R_{u i}}\right)$ & $b_{r i}[\Omega]$ & $R_{s i}$ \\
$a_{e i 1}\left[\frac{1}{s}\right]$ & $\left(\frac{1}{R_{i 1} C_{i 1}}\right)$ & $\alpha_{i}[$ Unitless $]$ & $R_{u i} \mathrm{c}_{\mathrm{f}}$ \\
$a_{e i 2}\left[\frac{1}{s}\right]$ & $\left(\frac{1}{R_{i 2} C_{i 2}}\right)$ & $\beta_{i}[$ Unitless] & $-1+\alpha_{i}$ \\
$b_{s i}\left[\frac{\Omega K}{W s}\right]$ & $\left(\frac{R_{s i}}{C_{s i}}\right)$ & $b_{e i 2}\left[\frac{V}{s A}\right]$ & $\left(\frac{1}{C_{i 2}}\right)$ \\
$b_{e i 1}\left[\frac{V}{s A}\right]$ & $\left(\frac{1}{C_{i 1}}\right)$ & $b_{e i 3}\left[\frac{1}{A s}\right]$ & $\left(\frac{1}{3600 C_{N i}}\right)$ \\
\hline
\end{tabular}

of battery $\mathrm{Cell}_{i}$ and $R_{u i}\left[K W^{-1}\right]$ is the convection thermal resistance for $\mathrm{Cell}_{i}$ and its value depends upon the geometry of the battery cell, coolant fluid properties and Nusselt number which in turn depends on Reynolds number. The coefficient $C_{s i}=\rho_{s i} c_{p s i} V_{s i}\left[\mathrm{JK}^{-1}\right]$ is the Heat Capacity where $\rho_{s i}$ is the density, $c_{p s i}$ is the Specific Heat Capacity and $V_{s i}\left[\mathrm{~m}^{3}\right]$ is the volume of $\mathrm{Cell}_{i}$. The coefficient $c_{f}=\rho_{f} c_{p f} \dot{V}_{f}\left[W K^{-1}\right]$ is the Thermal Conductance of the coolant fluid. All other quantities are shown in Figure 3.

In this paper, only one submodule (of a battery module), that consists of $n$ series connected battery cells, is studied. The thermal model is derived first separately for coolant flow in each direction and then two models are combined later to write the model for reciprocating air flow. In this study, both reciprocating and unidirectional air flow (UF ) are investigated and compared so for ease of reference the case of forward flow (i.e. from lower to higher cell index) is designated as UF . The thermal model proposed in [8] does not consider any power electronic switching of battery cells, so it must be adapted to the current framework. Thus, it is modified by embedding the switching function $s_{i}(t)$ and then it is combined with the enhanced Thevenin equivalent electrical model shown in Figure 3 to derive the switching electro-thermal model of a $\mathrm{PC}_{i}$ as follows. Assuming first the forward flow, the dynamics of the surface temperature $T_{s i}[K]$ of the battery Cell $_{i}$ in terms of $i_{L}(t)$ and $s_{i}(t)$, after substituting the value of $i_{B i}(t)$ from (1) into the model proposed in [8], is given by:

$$
\dot{T}_{s i}=-a_{s i} T_{s i}+b_{s i} i_{L}^{2} s_{i}^{2}+a_{s i} T_{f i-1}, \quad \forall i=\{1, \cdots, n\}
$$

where the term $i_{L}^{2} s_{i}^{2}$ represents the instantaneous ohmic power losses on the Cell $_{i}$ and $T_{f i-1}[K]$ is the temperature of temperature-node ' $i-1$ ' (of fluid element modeled using FNM) attached to $\mathrm{Cell}_{i}$ in upstream direction. According to [8], the temperatures of temperature-node ' $i-1$ ' and ' $i$ ' are related by:

$$
T_{f i}=\frac{\left(T_{s i}+\beta_{i} T_{f i-1}\right)}{\alpha_{i}}, \forall i
$$

By a forward recursion of equation (4), any $T_{f i}$ can be expressed as a function of inlet fluid temperature $T_{f 0}$ and the temperatures $T_{s 1}$ to $T_{s i}$ of battery cells. Thus:

$$
T_{f i}=a_{f i 1}^{(1)} T_{s 1}+a_{f i 2}^{(1)} T_{s 2}+\cdots+a_{f i i}^{(1)} T_{s i}+b_{f i}^{(1)} T_{f 0}
$$

where:

$$
\begin{aligned}
& a_{f i i}^{(1)}=\left(\frac{1}{\alpha_{i}}\right), \quad b_{f i}^{(1)}=\left(\frac{\prod_{k=1}^{i} \beta_{k}}{\prod_{k=1}^{i} \alpha_{k}}\right), \quad \forall i \geq 1 \\
& a_{f i j}^{(1)}=\left(\frac{\prod_{k=(j+1)}^{i} \beta_{k}}{\prod_{k=j}^{i} \alpha_{k}}\right), \forall i>j, \quad a_{f i j}^{(1)}=0, \quad \forall i<j
\end{aligned}
$$

Now using the expression (5) in (3), the thermal dynamics of battery cells can be re-written as follows:

$$
\dot{T}_{s i}=a_{t i 1}^{(1)} T_{s 1}+\cdots+a_{t i n}^{(1)} T_{s n}+b_{s i} i_{L}^{2} s_{i}^{2}+b_{t i}^{(1)} T_{f 0}
$$

where:

$$
\begin{aligned}
a_{t i i}^{(1)} & =-a_{s i}, \quad \forall i \geq 1 \\
a_{t i j}^{(1)} & =\left(\frac{\prod_{k=(j+1)}^{(i-1)} \beta_{k}}{\prod_{k=j}^{(i-1)} \alpha_{k}}\right) a_{s i}, \quad \forall i>j, \quad a_{t i j}^{(1)}=0, \forall i<j \\
b_{t i}^{(1)} & =\left(\frac{\prod_{k=1}^{(i-1)} \beta_{k}}{\prod_{k=1}^{(i-1)} \alpha_{k}}\right) a_{s i}, \quad \forall i \geq 1
\end{aligned}
$$

Analogous to forward flow case, the thermal dynamics of the battery $\mathrm{Cell}_{i}$ is derived for reverse coolant flow ( i.e. from higher to lower cell index) and the result is given below:

$$
\dot{T}_{s i}=a_{t i 1}^{(2)} T_{s 1}+\cdots+a_{t i n}^{(2)} T_{s n}+b_{s i} i_{L}^{2} s_{i}^{2}+b_{t i}^{(2)} T_{f n}
$$

where $T_{f n}$ is the temperature of inlet fluid entering the BSM from Cell $_{n}$ side and other coefficients are defined as follows:

$$
\begin{aligned}
& a_{t i i}^{(2)}=a_{t i i}^{(1)}, \quad \forall i \geq 1, \quad b_{t i}^{(2)}=b_{t(n-i+1)}^{(1)}, \quad \forall i \geq 1 \\
& a_{t i j}^{(2)}=a_{t j i}^{(1)}, \quad \forall i<j, \quad a_{t i j}^{(2)}=0, \quad \forall i>j
\end{aligned}
$$

The electrical equivalent model of a battery cell is shown in Figure 3. It is an enhanced Thevenin Model with two time 
constant behavior, see [20], [21], [22]. The dynamic model for this circuit is given by

$$
\begin{aligned}
\dot{V}_{i 1} & =-a_{e i 1} V_{i 1}+b_{e i 1} i_{L} s_{i}, \\
\dot{V}_{i 2} & =-a_{e i 2} V_{i 2}+b_{e i 2} i_{L} s_{i}, \\
\dot{\xi}_{i} & =-b_{e i 3} i_{L} s_{i} \\
V_{B i} & =f\left(\xi_{i}\right)-V_{i 1}-V_{i 2}-b_{r i} i_{L} s_{i}
\end{aligned}
$$

where $i_{B i}$ is the current flowing through the $\mathrm{Cell}_{i}$ and $\xi_{i}$ is the normalized state-of-charge (SoC) of Cell ${ }_{i}$. Note that $\xi_{i} \in[0,1]$ is a unit-less quantity. The $V_{i 1}$ and $V_{i 2}$ are the voltages across capacitors $C_{i 1}$ and $C_{i 2}$ respectively and $V_{B i}$ is the output voltage of $\mathrm{Cell}_{i}$. The SoC dependent open circuit voltage is given by $V_{\text {oci }}=f\left(\xi_{i}\right)$ where $f:[0,1] \rightarrow \mathbb{R}_{0}^{+}$is a function of SoC. Note that equations (8)-(18) describe the switched behavior of battery, under the switching action of the MLC, in terms of load current $i_{L}(t)$ and the switching function $s_{i}(t)$ and therefore we call it a switching model of a power cell $\mathrm{PC}_{i}$.

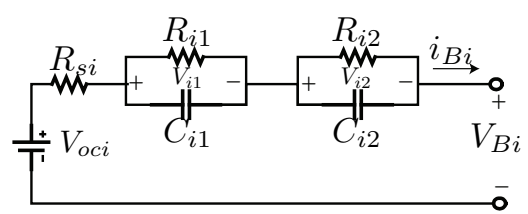

Fig. 3. Electrical model of a battery cell.

\section{B. Averaged-State-Space Model of a Power Cell}

In this study the aim is to evaluate the $O P$ and for that a model with real-valued control signal, which is far easier to handle in optimization problem compared to the case of discrete-valued signals, is needed. Since the switching model (8)-(18) involves discrete-valued signals $s_{i}(t)$, it is required to transform these signals to real-valued, averaged signals and modify the system model accordingly. The justification for use of averaging comes from the fact that, in most cases, the switching frequency $F_{s}$ inside the modulator $\mathcal{M}$ is much higher than the bandwidth $f_{L}$ of the system. So under the assumption $F_{s} \gg f_{L}$ and employing the two-time scale separation principle [23], the concept of averaging can be employed, see [24] and [25]. In other words it is assumed that the system response is determined predominantly by the duty cycle $u_{i}(t) \in[-1,1]$ i.e., the average of the switching input function $s_{i}(t)$. The following assumptions are made for derivation of the average quantities:

Assumption 1: The switching function can only attain values either from the set $\{0,1\}$ or $\{0,-1\}$ during any switching cycle of period $T_{s}$. This assumption implies that it is not allowed to charge and discharge the battery cell during the same switching cycle.

Assumption 2: The load current $i_{L}(t)$ remains fairly constant during any switching cycle. This assumption is justified based on the discussion above.

Assumption 3: All the internal electrical states $V_{i 1}=$ $\bar{V}_{i 1}, V_{i 2}=\bar{V}_{i 2}$ and $\xi_{i}=\bar{\xi}_{i}$ and the terminal voltage $V_{B i}$ remains fairly constant during the switching cycle.
Based on these assumptions, the average of the switching function $s_{i}(t)$ also called the duty-cycle is given by:

$$
u_{i}(t)=\bar{s}_{i}(t)=\frac{1}{T_{s}} \int_{t-T_{s}}^{t} s_{i}(t) \mathrm{d} t= \pm \frac{T_{o n}}{T_{s}}
$$

where $T_{\text {on }}$ is the ON time of a switch during any switching interval. It can be clearly seen from (19) that depending on the value of $T_{o n}, u_{i}(t)$ can attain any continuous real value in the interval $[-1,1]$. Now all other averaged signals can be defined in terms of $u_{i}(t)$ and $i_{L}(t)$ as follows:

$$
\begin{aligned}
& \bar{i}_{B i}(t)=u_{i} i_{L}, \quad i_{B r_{i}}^{2}=\left|u_{i}\right| i_{L}^{2} \\
& \bar{v}_{L i}=\left(f\left(\bar{\xi}_{i}\right)-\bar{V}_{i 1}-\bar{V}_{i 2}\right) u_{i}-b_{r i}\left|u_{i}\right| i_{L}
\end{aligned}
$$

where $\bar{i}_{B i}$ is the average current flowing through Cell ${ }_{i}$ during interval $T_{s}, i_{B r_{i}}$ is the root-mean-square (RMS) current that incurs equivalent ohmic loss across $\mathrm{Cell}_{i}$ over any switching cycle and $\bar{v}_{L i}$ is the average output voltage from $\mathrm{PC}_{i}$ during period $T_{s}$ of any switching cycle. See [18] for the detailed derivation of all the averaged variables. Now using these averaged quantities, the averaged-model of $\mathrm{PC}_{i}$ can be written as follows:

$$
\begin{aligned}
\dot{\bar{T}}_{s i} & =a_{t i 1}^{(\sigma)} \bar{T}_{s 1}+\cdots+a_{t i n}^{(\sigma)} \bar{T}_{s n}+b_{s i} i_{L}^{2}\left|u_{i}\right|+b_{t i}^{(\sigma)} T_{f \text { in }} \\
\dot{\bar{V}}_{i 1} & =-a_{e i 1} \bar{V}_{i 1}+b_{e i 1} i_{L} u_{i} \\
\dot{\bar{V}}_{i 2} & =-a_{e i 2} \bar{V}_{i 2}+b_{e i 2} i_{L} u_{i} \\
\dot{\bar{\xi}}_{i} & =-b_{e i 3} i_{L} u_{i} \\
\bar{V}_{B i} & =f\left(\bar{\xi}_{i}\right)-\bar{V}_{i 1}-\bar{V}_{i 2}-b_{r i} i_{L} u_{i}
\end{aligned}
$$

where $\sigma=1$ for forward and $\sigma=2$ for reverse coolant flow and $T_{f \text { in }} \in\left\{T_{f 0}, T_{f n}\right\}$ is the known fluid temperature at one of the two inlets depending on the direction of coolant flow. Since $\left|u_{i}\right|$ in (22) is not continuously differentiable, we define $u_{i}$ and $\left|u_{i}\right|$ in terms of two new control variables $u_{i 1}$ and $u_{i 2}$ which are defined as: $u_{i 1}=\max \left\{0, u_{i}\right\} \in[0,1]$ and $u_{i 2}=\max \left\{0,-u_{i}\right\} \in[0,1]$. Now we can write $u_{i}=\left(u_{i 1}-u_{i 2}\right) \in[-1,1]$ and $\left|u_{i}\right|=\left(u_{i 1}+u_{i 2}\right) \in[0,1]$. Note that $u_{i 1}$ can now be interpreted as duty cycle for Mode1 whereas $u_{i 2}$ can be interpreted as duty cycle for Mode2. In this new context, $u_{i 1}$ and $u_{i 2}$ can not be non-zero simultaneously (cf. assumption 1) at any time instant due to safety reasons which if violated can cause shoot-through. Thus in terms of newly defined control signal, the thermal subsystem of battery $\mathrm{Cell}_{i}$, for reciprocating coolant flow is given by:

$$
\dot{X}_{t i}=a_{t i 1}^{(\sigma)} X_{t 1}+\cdots+a_{t i n}^{(\sigma)} X_{t n}+\hat{g}_{t i}\left(x_{L}\right) \hat{u}_{i}+b_{t i}^{(\sigma)} T_{f \text { in }}
$$

where $X_{t i}=\bar{T}_{s i} \in \mathbb{R}, \hat{g}_{t i}\left(x_{L}\right)=\left[\begin{array}{ll}b_{s i} x_{L}^{2} & b_{s i} x_{L}^{2}\end{array}\right], \quad \hat{u}_{i}=$ $\left[\begin{array}{ll}u_{i 1} & u_{i 2}\end{array}\right]^{T} \in \mathbb{R}^{2}$ and $x_{L}=i_{L}$. Similarly, the electrical subsystem of battery $\mathrm{Cell}_{i}$ is given by:

$$
\dot{X}_{e i}=A_{e i} X_{e i}+\hat{g}_{e i}\left(x_{L}\right) \hat{u}_{i}
$$

where $X_{e i}=\left[\begin{array}{lll}X_{e i 1} & X_{e i 2} & X_{e i 3}\end{array}\right]^{T} \in \mathbb{R}^{3}$ with $X_{e i 1}=\bar{V}_{i 1}, X_{e i 2}=\bar{V}_{i 2}, X_{e i 3}=\bar{\xi}_{i}$ and $A_{e i}=\operatorname{diag}\left(-a_{e i 1},-a_{e i 2}, 0\right) \in \mathbb{R}^{3 \times 3}, \hat{g}_{e i}\left(x_{L}\right)=$ $\left[\begin{array}{ll}b_{e i} x_{L} & -b_{e i} x_{L}\end{array}\right] \in \mathbb{R}^{3 \times 2}$ with $b_{e i}=\left[\begin{array}{lll}b_{e i 1} & b_{e i 2} & -b_{e i 3}\end{array}\right]^{T}$. 


\section{Complete Averaged State-Space Model of n-Cell MLC}

There are various possible state-space representations for a $n$-cell MLC depending on number of cells and the configuration in which they are connected inside each $\mathrm{PC}_{i}$. Here it is assumed that each $\mathrm{PC}_{i}$ contains only one Cell $i$ so using (27) and (28) as basic building block, the state-space system for thermal subsystem of $n$ cells can be written as follows:

$$
\dot{X}_{t}=A_{t}^{(\sigma)} X_{t}+\hat{G}_{t}\left(x_{L}\right) \hat{u}+W_{t}^{(\sigma)} T_{f \mathrm{in}}, \quad Y=C_{t} X_{t}
$$

Here $A_{t}^{(\sigma)} \in \mathbb{R}^{n \times n}$ is a system matrix where $A_{t}^{(1)}$ is a lower triangular matrix with coefficients $a_{t i j}^{(1)}$ defined by (9) and (10) for forward coolant flow and $A_{t}^{(2)}=\left(A_{t}^{(1)}\right)^{T}$ is an upper triangular matrix with coefficients $a_{t i j}^{(2)}$ defined by (13) and (14) for reverse coolant flow. $\hat{G}_{t}\left(x_{L}\right)=$ $\operatorname{diag}\left(\hat{g}_{t 1}\left(x_{L}\right), \cdots, \hat{g}_{t n}\left(x_{L}\right)\right) \in \mathbb{R}^{n \times 2 n}$ is a load currentdependent input matrix for thermal subsystem, $W_{t}^{(\sigma)}=$ $\left[\begin{array}{lll}b_{t 1}^{(\sigma)} & \cdots & b_{t n}^{(\sigma)}\end{array}\right]^{T} \in \mathbb{R}^{n}$, with coefficients $b_{t i}^{(\sigma)}$ defined by (11) for $\sigma=1$ and (13) for $\sigma=2$, is the scaling vector for the inlet fluid temperature and $C_{t}=I_{n} \in \mathbb{R}^{n \times n}$ is an output matrix, $X_{t}=\left[\begin{array}{lll}X_{t 1} & \cdots & X_{t n}\end{array}\right]^{T} \in \mathbb{R}^{n}$ is a thermal state vector, $\hat{u}=\left[\begin{array}{lll}\hat{u}_{1}^{T} & \cdots & \hat{u}_{n}^{T}\end{array}\right]^{T} \in \mathbb{R}^{2 n}$ is the input vector, $T_{f \text { in }} \in \mathbb{R}$ is the known fluid temperature $\left(T_{f 0}\right.$ or $\left.T_{f n}\right)$ at one of the two inlets depending on the direction of coolant flow and $Y \in \mathbb{R}^{n}$ is an output vector. Similarly the electrical subsystem of $n$-cells is given by:

$$
\dot{X}_{e}=A_{e} X_{e}+\hat{G}_{e}\left(x_{L}\right) \hat{u}
$$

Here $A_{e}=\operatorname{diag}\left(A_{e 1}, \cdots, A_{e n}\right) \in \mathbb{R}^{3 n \times 3 n}$ is a system matrix and $\hat{G}_{e}\left(x_{L}\right)=\operatorname{diag}\left(\hat{g}_{e 1}\left(x_{L}\right), \cdots, \hat{g}_{e n}\left(x_{L}\right)\right) \in \mathbb{R}^{3 n \times 2 n}$ is a load current-dependent input matrix for electrical subsystem, $X_{e}=\left[\begin{array}{lll}X_{e 1}^{T} & \cdots & X_{e n}^{T}\end{array}\right]^{T} \in \mathbb{R}^{3 n}$ is an electrical state vector, $\hat{u} \in \mathbb{R}^{2 n}$ is the input vector. Now the two subsystems can be combined in diagonal form:

$$
\begin{gathered}
\underbrace{\left[\begin{array}{c}
\dot{X}_{t} \\
\dot{X}_{e}
\end{array}\right]}_{\dot{X}}=\underbrace{\left[\begin{array}{cc}
A_{t}^{(\sigma)} & 0 \\
0 & A_{e}
\end{array}\right]}_{A^{(\sigma)}} \underbrace{\left[\begin{array}{c}
X_{t} \\
X_{e}
\end{array}\right]}_{X}+\underbrace{\left[\begin{array}{c}
\hat{G}_{t}\left(x_{L}\right) \\
\hat{G}_{e}\left(x_{L}\right)
\end{array}\right]}_{\hat{G}\left(x_{L}\right)} \hat{u}+\underbrace{\left[\begin{array}{c}
W_{t}^{(\sigma)} \\
0
\end{array}\right]}_{W^{(\sigma)}} T_{f \text { in }} \\
\dot{X}=A^{(\sigma)} X+\hat{G}\left(x_{L}\right) \hat{u}+W^{(\sigma)} T_{f \text { in }}, \quad Y=C X
\end{gathered}
$$

where $A^{(\sigma)} \in \mathbb{R}^{4 n \times 4 n}$ is a system matrix, $\hat{G}\left(x_{L}\right) \in \mathbb{R}^{4 n \times 2 n}$ is a load current-dependent input matrix for complete system, $C=\left[\begin{array}{ll}C_{t} & 0\end{array}\right] \in \mathbb{R}^{n \times 4 n}$ is the output matrix. $X \in \mathbb{R}^{4 n}$ is a state vector, $\hat{u} \in \mathbb{R}^{2 n}$ is the input vector and $W^{(\sigma)} \in \mathbb{R}^{4 n}$ is the scaling vector for the inlet fluid temperature. Note that the average state-space electro-thermal model under reciprocating air flow as shown in (31) is a piecewise affine (PWA) system.

\section{Optimization PROBlem}

In this section, the optimization problem is formulated for $O P$ scheme to achieve cell balancing in terms of both temperature and SoC. The averaged state-space model derived in previous section is used along with an objective function and some constraints as described below.

\section{A. Definition of Objective Function}

The objective is to equalize the SoC of all cells at the final time and keep both the SoC and temperature deviations among the cells within a certain zone during the whole drive cycle. These objectives will be specified as constraints in the next subsection. In addition to this, the aim is to minimize the temperature deviations among battery cells which is specified here as the following objective function:

$$
J(Y)=\int_{0}^{t_{f}}\left(Y_{1}-Y_{2}\right)^{2}+\cdots+\left(Y_{n-1}-Y_{n}\right)^{2} d t
$$

To bring $J(Y)$ on the quadratic form in $X$, let's define $Q=$ $C^{T} \bar{Q}_{1} \bar{Q}_{1}^{T} C$ with $\bar{Q}_{1}=\operatorname{diag}\left(q_{1}, \cdots, q_{n-1}\right) \in \mathbb{R}^{n \times(n-1)}$ where $q_{i}=\left[\begin{array}{ll}1 & -1\end{array}\right]^{T}$. Now the objective function (32) can be rewritten on the following standard quadratic form:

$$
J(X)=\int_{0}^{t_{f}} X^{T} Q X d t
$$

\section{B. Definition of Constraints}

During run-time we want SoC of all cells to stay within a certain zone from each other given by:

$$
\begin{aligned}
& -\Delta S o C \leq\left(X_{e i 3}(t)-X_{e j 3}(t)\right) \leq \Delta S o C, \\
& \forall t, \forall i, j \in\{1,2, \cdots, n\}
\end{aligned}
$$

and at final time the SoC of all cells should be equal:

$$
X_{e i 3}\left(t_{f}\right)=X_{e j 3}\left(t_{f}\right) \quad \forall i, j \in\{1,2, \cdots, n\}
$$

Also the SoC of each $\mathrm{Cell}_{i}$ must stay within following zone:

$$
0 \leq X_{e i 3}(t) \leq 1 \quad \forall t, \forall i \in\{1,2, \cdots, n\}
$$

To ensure tight thermal balancing, in addition to minimizing the deviations of cell temperatures, there is a hard constraint to keep temperature deviations among the cells in the following zone:

$$
-\Delta T_{s} \leq\left(T_{s i}(t)-T_{s j}(t)\right) \leq \Delta T_{s} \quad \forall t, \forall i, j \in\{1, \cdots, n\}
$$

Moreover, there is a safety constraint on the maximum operating temperature of each cell:

$$
T_{s i}(t) \leq T_{\text {smax }} \forall t, \forall i \in\{1,2, \cdots, n\}
$$

where $T_{\text {smax }}$ is the maximum operating temperature allowed for each $\mathrm{Cell}_{i}$. The objective to track demanded load voltage $\left(v_{L d}\right)$ can be written as the following constraint:

$$
v_{L d}=\sum_{i=1}^{n}\left[\left(f\left(X_{e i 3}\right)-X_{e i 1}-X_{e i 2}\right) u_{i}-b_{r i}\left|u_{i}\right| x_{L}\right]
$$

The $v_{L d}$ is normally provided by the higher supervisory block called Energy Management System (EMS) in context of Hybrid Electric Vehicles (HEV). The $u_{i}=\left[\begin{array}{ll}1 & -1\end{array}\right] \hat{u}_{i}$ is the duty cycle of $\mathrm{Cell}_{i}$. In this study it is assumed that $f\left(X_{e i 3}\right)$ is constant and $X_{e i 1}$ and $X_{e i 2}$ are negligible which is a normal assumption used for developing the EMS for (P)HEVs, see [26]. These assumptions are being made to preserve the convexity of the problem. There is a constraint 
on the maximum current as well that each battery cell can supply:

$$
x_{L} u_{i} \in\left[\bar{i}_{\text {Bimin }}, \bar{i}_{\text {Bimax }}\right]
$$

where $\bar{i}_{\text {Bimin }}$ and $\bar{i}_{\text {Bimax }}$ are, respectively, minimum and maximum battery current limits. There are some constraints on the control signal $\hat{u}_{i}=\left[\begin{array}{ll}u_{i 1} & u_{i 2}\end{array}\right]^{T} \in \mathbb{R}^{2}$ given by:

$$
\begin{aligned}
& u_{i 1} \in[0,1], \quad u_{i 2} \in[0,1], \quad\left|u_{i}\right|=\left(u_{i 1}+u_{i 2}\right) \in[0,1], \\
& \text { and } u_{i}=\left(u_{i 1}-u_{i 2}\right) \in[-1,1]
\end{aligned}
$$

As per the definition of $u_{i 1}$ and $u_{i 2}$ given in previous section, they cannot be nonzero simultaneously due to shoot-through problem so to ensure the safety, the following constraint is imposed:

$$
u_{i 1} u_{i 2}=0
$$

Note that the last constraint is non-convex and we need to get rid of it in order to preserve convexity of the problem.

\section{Definition of Optimization Problem}

Now we can write an optimization problem as follows:

$$
\begin{aligned}
& J^{0}=\min _{\hat{u}} \int_{0}^{t_{f}} X^{T} Q X \mathrm{~d} t \quad \text { subject to } \\
& \left\{\begin{array}{l}
\dot{X}=A^{(\sigma)} X+\hat{G}\left(x_{L}\right) \hat{u}+W^{(\sigma)} T_{f \text { in }}, \\
\text { Constraints }(34)-(42), \\
x_{L}(t), T_{f \text { in }} \text { and } \sigma \text { are known at each time step. }
\end{array}\right.
\end{aligned}
$$

The optimization problem (P-I) is non-convex due to nonconvex constraint $u_{i 1} u_{i 2}=0$. In the next subsection, some assumptions are made to restore the convexity and simplify the problem.

\section{Solution of Optimization Problem Using CVX}

To solve problem (P-I) we used CVX, a MATLAB-based package for specifying and solving convex programs, see [27], [28], using disciplined convex programming ruleset, see [29]. Before setting up the optimization problem (P-I) in CVX, the non-convex constraint $\left(u_{i 1} u_{i 2}=0\right)$ need to be removed. This is done by following the approach similar to that in [18]. In short, it is not allowed at any time instant to charge any cell while discharging others. Therefore, using this assumption, the sign of $u_{i}$ can be pre-decided based on the sign of known demanded load voltage $\left(v_{L d}\right)$. Thus, the non-convex constraint $\left(u_{i 1} u_{i 2}=0\right)$ need not to be specified. The system has been discretized using Euler's approximation with sampling time $h=1 \mathrm{sec}$. The simulation parameters are shown in table II where $\bar{R}_{s}$ is the nominal value of series resistance, $R_{s i}$, of any $\operatorname{Cell}_{i}, N$ is the prediction (or driving) horizon in discrete time and $\tau$ is the reciprocation period i.e., the period in which coolant completes one cycle of uniform forward and reverse flow.

\section{Simulation Results}

Before presenting the simulation results, we introduce some new variables which can be illustrated in plots with more clarity. Let us define the average temperature $\bar{X}_{t i}=$ $\frac{1}{N} \sum_{k=0}^{N} X_{t i}(k)$ of each Cell $_{i}$ over the whole driving horizon
TABLE II

SIMULATION PARAMETERS

\begin{tabular}{lc|lc}
\hline Parameters & Value & Parameters & Value \\
\hline$n$ & 5 & $\bar{i}_{\text {Bimin }}$ & $-200 \mathrm{~A}, \forall i$ \\
$t_{f}$ & $12 \mathrm{~min}$ & $\bar{i}_{\text {Bimax }}$ & $200 \mathrm{~A}, \forall i$ \\
$h$ & $1 \mathrm{sec}$ & $\Delta T_{s}$ & $2{ }^{\circ} \mathrm{C}$ \\
$N$ & $t_{f} / h=720$ & $\Delta S o \mathrm{C}$ & 0.1 \\
$\bar{R}_{s}$ & $6.2770 \mathrm{~m} \Omega$ & $T_{\text {smax }}$ & $40^{\circ} \mathrm{C}$ \\
$R_{s 5}$ & $1.48 \bar{R}_{s}=9.29 \mathrm{~m} \Omega$ & $T_{\text {fin }}$ & $20^{\circ} \mathrm{C}$ \\
$T_{s i}(0)$ & $25^{\circ} \mathrm{C}, \forall i$ & $\tau$ & $60 \mathrm{sec}$ \\
\hline
\end{tabular}

$N$ and the average temperature of the battery submodule given by $\bar{X}_{t b}=\frac{1}{n} \sum_{i=1}^{n} \bar{X}_{t i}$. Similarly the normalized average power loss per unit ohm across any $\mathrm{Cell}_{i}$ over the whole driving horizon is given by:

$$
\bar{I}_{i}=\frac{\frac{1}{N} \sum_{k=1}^{N} i_{B r_{i}}^{2}(k)}{\max _{j}\left(\left(i_{B r_{j}}^{o u}\right)^{2}\right)}
$$

where $i_{B r_{j}}^{o u}$ as given in (20) is RMS current through Cell ${ }_{j}$ for $O P$ under $U F$. Note that to differentiate between signals of $O P$ and UDCO the corresponding ' $O$ ' and ' $u$ ' superscripts are used along with ' $u$ ' and ' $r$ ' to designate UF and $\mathrm{RF}$ respectively. Now we are ready to present simulation results below.

\section{A. Battery States as a function of time: OP Versus UDCO}

In this subsection we evaluate the performance of UDCO and $\mathrm{OP}$ under both $\mathrm{UF}$ and RF . Here it is assumed that the Cell 5 has almost $50 \%$ higher series resistance due to aging or some other effect. The temperature $\left(X_{t i}\right)$, SoC $\left(\xi_{i}\right)$ and the normalized average per unit power loss $\left(\bar{I}_{i}\right)$ are plotted for each cell. Simulation results are shown in Figure 4 for both $\mathrm{OP}$ and UDCO under RF as well as UF. Figure 4(a) shows the drive cycle data i.e. demanded power, voltage and current profiles. The simulation results for $\mathrm{RF}$ are shown in Figures 4(b)-4(e) and those for UF are shown in Figures 4(f)-4(h).

Reciprocating Air Flow: Figure 4(b) shows temperatures of all cells for $O P$ and Figure 4(c) for UDCO policy under RF. It is clearly seen that temperature of $\mathrm{Cell}_{5}$ using UDCO policy is significantly higher even under $R F$ compared to that of $\mathrm{OP}$. Moreover OP has achieved good thermal balancing while keeping temperatures of all cells within $\pm 2{ }^{\circ} \mathrm{C}$ zone and SoC within $\pm 10 \%$ from each other as shown in Figure 4(d). Figure 4(e) shows the normalized average unit power loss $\bar{I}_{i}$ for each cell. The horizontal dashed black line shows the average unit power loss $\bar{I}_{i}^{u r}$ across each cell for UDCO policy and colored vertical bars show the average unit power loss $\bar{I}_{i}^{\text {or }}$ across each cell for $\mathrm{OP}$ scheme. The internal resistance $R_{s 5}$ of $\mathrm{Cell}_{5}$ is almost $50 \%$ higher than other cells. Thus, as shown in Figure 4(e), the naturally optimal policy is to use $\mathrm{Cell}_{5}$ less compared to others and $\mathrm{Cell}_{1}$, which is in the best thermal condition, should be used most. It is also pertinent to mention here that $\mathrm{OP}$ achieves the thermal balancing by deciding to use $\mathrm{Cell}_{5}$ less compared to other cells during high current intervals and thus the $\mathrm{Cell}_{5}$ sees less ohmic losses. This policy is naturally optimal as losses are quadratic in current. 
Unidirectional Flow: Figure 4(f) shows temperatures of all cells for OP, Figure 4(g) for UDCO policy, Figure 4(h) shows the normalized average unit power loss for each cell under UF. Once again, as shown in figures, the OP policy performs better than UDCO. Similar to RF case, OP has simultaneously achieved good thermal and SoC balancing. For brevity, SoC plot is not shown here but it is almost same as for RF case. See [18] for more detailed comparison between $\mathrm{OP}$ and UDCO under UF .

\section{B. Unidirectional Versus Reciprocating Air Flow}

In this section UF and RF are compared for both $\mathrm{OP}$ and UDCO . Temperature of cells for OP policy under UF is shown in Figure 4(f) and that for $O P$ under RF in Figure 4(b). These figures clearly show that when using $O P$ there is not any significant gain from $\mathrm{RF}$ especially for short series-connected battery string. Similarly temperatures of cells for UDCO under $\mathrm{UF}$ is shown in Figure 4(g) and that for UDCO under RF is shown in Figure 4(c). As shown, though RF with UDCO policy has helped to minimize temperature deviations among cells with nominal resistance $\left(\mathrm{Cell}_{1} \cdots \mathrm{Cell}_{4}\right)$ but it is not that useful for $\mathrm{Cell}_{5}$ that has $50 \%$ higher resistance. Also note the difference between average unit power loss in UF and $\mathrm{RF}$ case as shown in Figures 4(h) and 4(e). Figure 4(i) shows the average temperature of BSM under four different control policies: 1) UDCO under RF 2) UDCO under UF 3) OP under $\mathrm{RF}$ and 4) $\mathrm{OP}$ under UF . This figure clearly shows that RF does not help in reducing the overall mean temperature of BSM as the average temperature is almost the same for all policies. The main purpose of $R F$ is just to achieve temperature uniformity but an important point to stress here is that the same can also be achieved even under UF by shifting the power losses between cells using the MLC-based active cell balancing system when operated using $\mathrm{OP}$. Moreover, RF does not give any significant help under resistance variations whereas $\mathrm{OP}$ handles this quite well. Thus $\mathrm{OP}$ in contrast to RF not only ensures temperature uniformity in nominal conditions but also under parameter variations.

\section{SUMMARY AND CONCLUSIONS}

This article has investigated the potential benefit of optimally using the extra degree-of-freedom (DoF) of multilevel converter (MLC) for simultaneous balancing of both stateof-charge (SoC) and temperature of cells under reciprocating air flow $(R F)$. The complete state-space electro-thermal model has been derived for RF and a constrained convex optimization problem has been formulated and solved based on the assumption that the state of each cell and the schedule of reciprocating air flow is perfectly known. The simulation results show that even for $50 \%$ increase in internal resistance of the downstream cell the $O P$ policy, that optimally uses the extra DoF of MLC, gives significant reduction in temperature deviation among cells compared to ad hoc uniform duty cycle operation. Moreover $\mathrm{OP}$ can also achieve the temperature uniformity, under parameter variations, even with UF whereas $\mathrm{RF}$ without $\mathrm{OP}$ cannot keep the temperature uniformity in such circumstances. This study indicates that when using MLCbased $O P$ there is not any significant advantage in using $R F$.
Thus, RF seems redundant function in the presence of MLCbased active cell balancing system when operated using $\mathrm{OP}$.

\section{ACKNOWLEDGMENTS}

The authors would like to thank Nikolce Murgovski, Oskar Josefsson, Torbjörn Thiringer and Anders Grauers for all the positive discussions while developing this work.

\section{REFERENCES}

[1] B. Kuhn, G. Pitel, and P. Krein, "Electrical properties and equalization of lithium-ion cells in automotive applications," in Vehicle Power and Propulsion, 2005 IEEE Conference, sept. 2005, p. 5 pp.

[2] S. Lukic, J. Cao, R. Bansal, F. Rodriguez, and A. Emadi, "Energy storage systems for automotive applications," Industrial Electronics, IEEE Transactions on, vol. 55, no. 6, pp. 2258 -2267, june 2008.

[3] K. Smith, T. Markel, K. Gi-Heon, and A. Pesaran, "Design of electric drive vehicle batteries for long life and low cost," Accelerated Stress Testing and Reliability (ASTR), IEE Workshop on, Oct. 6-8 2010.

[4] C. Park and A. Jaura, "Reciprocating battery cooling for hybrid and fuel cell vehicles," ASME International Mechanical Engineering Congress and Exposition (IMECE2003), Washington, DC, USA, pp. 425-430, november 2003

[5] _ - "Dynamic thermal model of Li-Ion battery for predictive behavior in hybrid and fuel cell vehicles," SAE transactions, vol. 112, no. 3, pp. 1835-1842, 2003.

[6] — "Transient heat transfer of $42 \mathrm{~V} \mathrm{Ni-MH}$ batteries for an HEV application," Future Car Congress, 2002.

[7] A. Pesaran and B. S. Vlahinos, A, "Thermal performance of EV and HEV battery modules and packs," Proceedings of the 14th International Electric Vehicle Symposium, Orlando, Florida, December 1997.

[8] R. Mahamud and C. Park, "Reciprocating air flow for li-ion battery thermal management to improve temperature uniformity," Journal of Power Sources, vol. 196, no. 13, pp. 5685 - 5696, 2011

[9] C. Motloch, J. Christophersen, J. Belt, R. Wright, G. Hunt, T. Tartamella, H. Haskins, and T. Miller, "High-Power Battery Testing Procedures and Analytical Methodologies for HEV's," SAE 2002-01-1950, 2002.

[10] W. C. Lee, D. Drury, and P. Mellor, "Comparison of passive cell balancing and active cell balancing for automotive batteries," in Vehicle Power and Propulsion Conference (VPPC), 2011 IEEE, sept. 2011, pp. $1-7$.

[11] J. Cao, N. Schofield, and A. Emadi, "Battery balancing methods: A comprehensive review," in Vehicle Power and Propulsion Conference, 2008. VPPC 'O8. IEEE, sept. 2008, pp. $1-6$.

[12] W. Bentley, "Cell balancing considerations for lithium-ion battery systems," in Battery Conference on Applications and Advances, 1997., 12th Annual, jan 1997, pp. 223 -226.

[13] P. Krein, "Battery management for maximum performance in plugin electric and hybrid vehicles," in Vehicle Power and Propulsion Conference, 2007. VPPC 2007. IEEE, sept. 2007, pp. 2 -5.

[14] J. Rodriguez, L. Franquelo, S. Kouro, J. Leon, R. Portillo, M. Prats, and M. Perez, "Multilevel converters: An enabling technology for highpower applications," Proceedings of the IEEE, vol. 97, no. 11, pp. 1786 -1817 , nov. 2009.

[15] M. Malinowski, K. Gopakumar, J. Rodriguez, and M. Pe andrez, "A survey on cascaded multilevel inverters," Industrial Electronics, IEEE Transactions on, vol. 57, no. 7, pp. 2197 -2206, july 2010.

[16] L. Tolbert, F. Z. Peng, and T. Habetler, "Multilevel converters for large electric drives," Industry Applications, IEEE Transactions on, vol. 35, no. 1 , pp. $36-44$, jan/feb 1999.

[17] O. Josefsson, A. Lindskog, S. Lundmark, and T. Thiringer, "Assessment of a multilevel converter for a phev charge and traction application," in Electrical Machines (ICEM), 2010 XIX International Conference on, sept. 2010, pp. $1-6$.

[18] F. Altaf, L. Johannesson, and B. Egardt, "Evaluating the potential for cell balancing using a cascaded multi-level converter using convex optimization," IFAC Workshop on Engine and Powertrain Control, Simulation and Modeling, Oct. 2012. To appear.

[19] N. Mohan, T. M. Undeland, and W. P. Robbins, Power Electronics: Converters, Applications, and Design, 3rd ed. John Wiley \& Sons; 2003, 2003.

[20] F. Codeca, S. Savaresi, and G. Rizzoni, "On battery state of charge estimation: A new mixed algorithm," in Control Applications, 2008. CCA 2008. IEEE International Conference on, sept. 2008, pp. 102 107. 


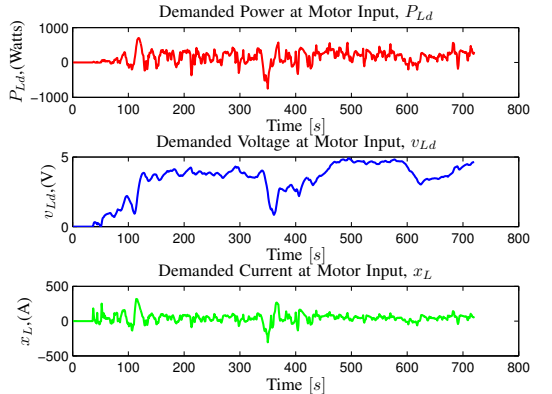

(a) Drive Cycle: Demanded Power, Voltage and Current Profile. Note that there are two short duration high power peaks: First around 120 seconds and second around 350 seconds.

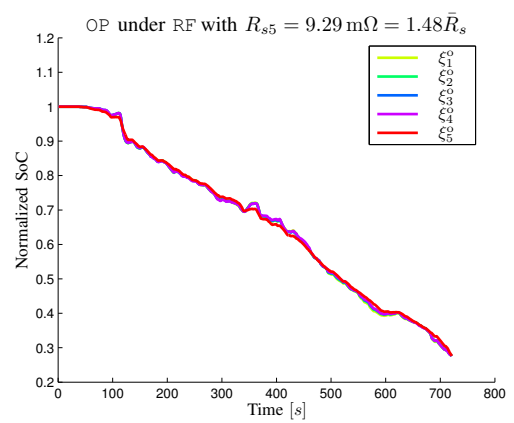

(d) Optimal SoC of each cell for OP under $\mathrm{RF}$. The plot shows that OP has simultaneously achieved the SoC balancing in addition to thermal balancing shown in Fig. 4(b).

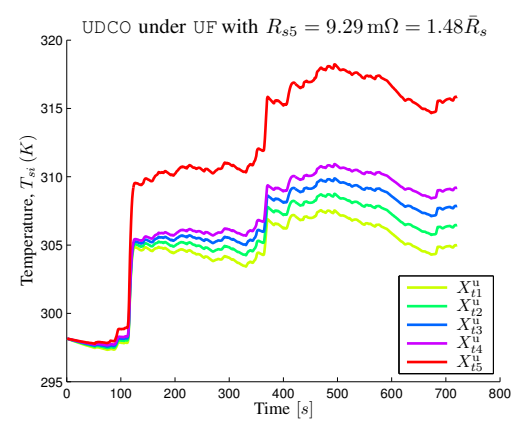

(g) Temperature of each cell for UDCO under UF . Red: Hottest Cell, Light Green: Coldest Cell. Cell 5 suffers from thermal run away as shown in red.

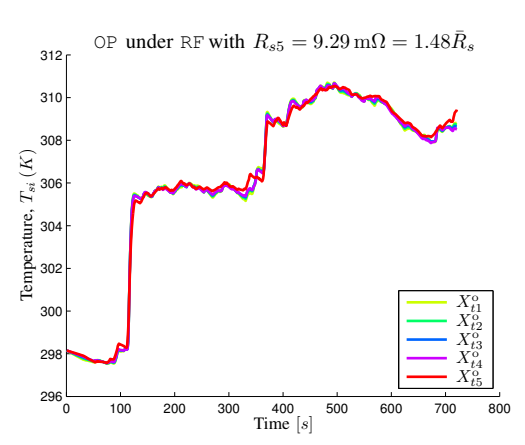

(b) Optimal Temperature of each cell for OP under RF. Despite $R_{s 5}$ being $50 \%$ higher, OP has successfully achieved thermal balancing among all cells.

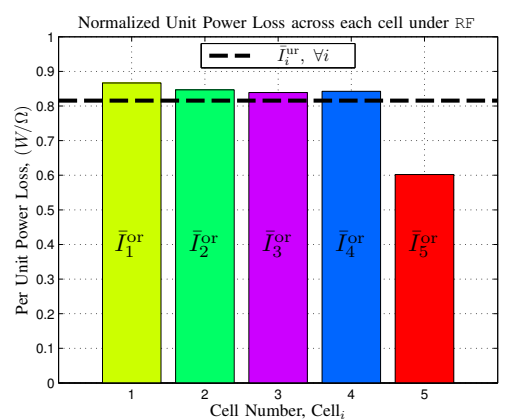

(e) Optimal normalized average power loss per unit ohm across each Cell ${ }_{i}$ for OP under $\mathrm{RF}$. The dashed horizontal black line shows normalized unit power loss across each $\mathrm{Cell}_{i}$ for UDCO .

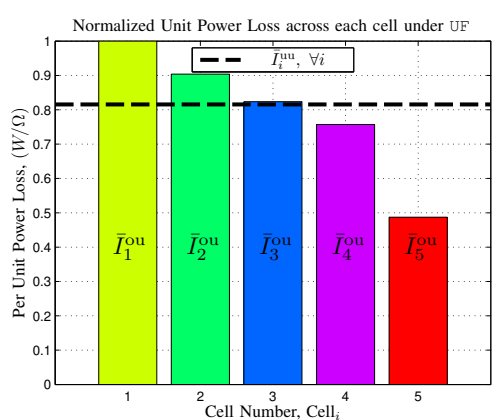

(h) Optimal normalized average power loss per unit ohm across each $\mathrm{Cell}_{i}$ for OP under UF. The dashed horizontal black line shows normalized unit power loss across each $\mathrm{Cell}_{i}$ for UDCO.

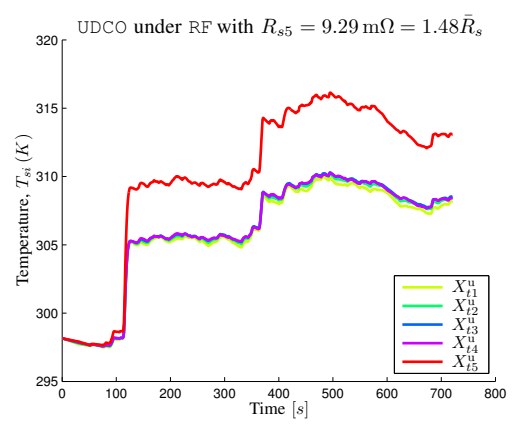

(c) Temperature of each cell for UDCO under RF . Red: Hottest Cell, Light Green: Coldest Cell. Cell 5 suffers from thermal run away as shown in red.

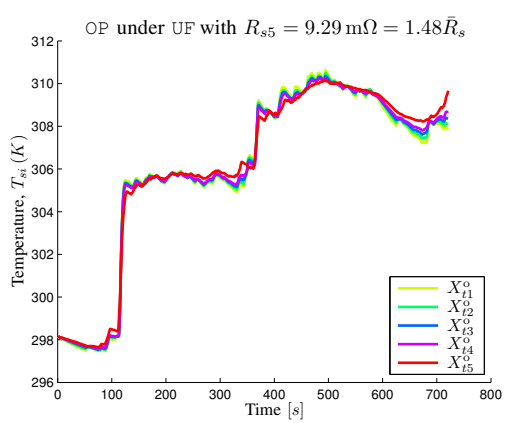

(f) Optimal Temperature of each cell for OP under UF. Despite $R_{s 5}$ being $50 \%$ higher, OP has successfully achieved thermal balancing among all cells.

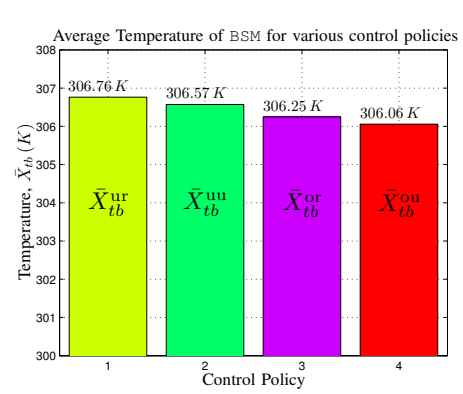

(i) Average temperature of BSM under various control policies: 1) UDCO under RF 2) UDCO under UF 3) OP under RF 4) OP under $\mathrm{UF}$. Average temperature is almost same for all policies.

Fig. 4. Simulation results and comparison between OP and UDCO for both UF and RF. The plots show that OP significantly performs better than UDCO under both UF and RF. The uniform use of cells is naturally not optimal in this situation since resistance $\left(R_{s 5}\right)$ of the Cell 5 is $50 \%$ higher than others. The plots also shows that using RF is not much helpful for UDCO in presence of parameter variation. Moreover OP exhibits similar performance under both UF and $\mathrm{RF}$ and thus RF is redundant for MLC-based active cell balancing system when operated using $\mathrm{OP}$.

[21] M. Chen and G. Rincon-Mora, "Accurate electrical battery mode capable of predicting runtime and i-v performance," Energy Conversion, IEEE Transactions on, vol. 21, no. 2, pp. 504 - 511, june 2006.

[22] H. He, R. Xiong, X. Zhang, F. Sun, and J. Fan, "State-of-charge estimation of the lithium-ion battery using an adaptive extended kalman filter based on an improved thevenin model," Vehicular Technology, IEEE Transactions on, vol. 60, no. 4, pp. 1461 -1469, may 2011.

[23] H. Khalil, Nonlinear systems. Prentice Hall, NJ, 2002.

[24] J. Kassakian, M. Schlecht, and G. Verghese, Principles of Power Electronics. Addison-Wesley, 1991.

[25] S. Sirisukprasert, "The Modeling and Control of a Cascaded-Multilevel Converter-Based STATCOM," PhD Thesis, Virginia Tech, 2004.
[26] L. Guzzella and A. Sciarretta, Vehicle Propulsion Systems. Springer, 2005.

[27] M. Grant and S. Boyd, "CVX: Matlab software for disciplined convex programming, version 1.21," ../../cvx, Apr. 2011.

[28] - "Graph implementations for nonsmooth convex programs," in Recent Advances in Learning and Control, ser. Lecture Notes in Control and Information Sciences, V. Blondel, S. Boyd, and H. Kimura, Eds. Springer-Verlag Limited, 2008, pp. 95-110, http://stanford.edu/ boyd/ graph_dcp.html.

[29] S. Boyd and L. Vandenberghe, Convex Optimization. Cambridge University Press, 2006. 